\title{
Exercise Effects on Gutdysbiosis, Intestinal Permeability and Systemic Inflammation in Patients with Type 2 Diabetes: A Pilot Study
}

\author{
Evasio Pasini ${ }^{1 *}$, Giovanni Corsetti ${ }^{2}$, Deodato Assanelli ${ }^{3}$, Cristian Testa $^{4}$, Claudia Romano $^{2}$, Francesco S Dioguardi ${ }^{5}$ \\ and Roberto Aquilani ${ }^{6}$ \\ ${ }^{1}$ Cardiac Rehabilitation Division, Maugeri Scientific Clinical Institutes, Italy \\ ${ }^{2}$ Division of Human Anatomy and Physiopathology, Department of Clinical and Experimental Sciences, University of Brescia, Italy
}

${ }^{3}$ Division of General Medicine, Azienda Ospedaliera Spedali Civili di Brescia, Italy

${ }^{4}$ Laboratory of clinical microbiology and virology Functional Point, Italy

${ }^{5}$ Department of Clinical Sciences and Community Health, University of Milan, Italy

${ }^{6}$ Department of Biology and Biotechnology, University of Pavia, Italy

Submission: November 20, 2017; Published: December 15, 2017

*Corresponding author: Evasio Pasini, Cardiac Rehabilitation Division, Maugeri Scientific Clinical Institutes, IRCCS Lumezzane, Lumezzane, Brescia, Italy, Tel: 0039-030-8253135; Email: evpasini@gmail.com

\begin{abstract}
Exercise plays a significant role in the prevention of the diabetes. Recent data propose that dysbiosis of intestinal microbiota composition contributes to development of Type 2 diabetes (T2D). Moreover, dysbiosis alters intestinal endothelium permeability causing the "Leaky gut syndrome" (LGS). We measured in 15 selected patients with standard medical cures for sable T2D the effects of 6 months of endurance, resistance and flexibility training on the gut microbiota composition and intestinal permeability. At baseline, T2D patients had high biochemical parameters (glycaemia, HOMA index, HbA1c, C-Reactive Protein [CRP]) with dysbiosis (elevated concentration of Mycetes) and altered intestinal impermeability (Measured by faecal Zonulin). Alter chronic exercise, glycaemia, HOMA index, HbA1c and CPR were reduced as well as faecal presence of Mycetesspp and Zonulin. This pilot study showed that selected patients with T2D had intestinal dysbiosis with overgrow of Mycetes, presence of LGS and low grade inflammation. Interestingly, chronic exercise significantly reduced all these parameters.
\end{abstract}

Keywords: Exercise; Diabetes; Microbiota; Dysbiosis; Zonulin; Leaky gut syndrome

\section{Introduction}

Evidences show that exercise plays a significant role in the prevention of the diabetes and control of glycaemia as well as in the diabetes-related organ complications [1]. Furthermore, recent data propose microbiota composition as possible potential environmental contributor to development of T2D [2]. Indeed, gut dysbiosis influences fundamental intestinal functions as epithelium permeability, causing the "Leaky gut syndrome" (LGS) [3]. As consequence, LGS heavily influences gut functions including digestive, absorptive and endocrine activities that, in turns, may influence glucose metabolism. Moreover, LGS activates inflammation allowing translocation of microorganisms from the intestinal lumen to the blood circulation. Interestingly, recent papers show that physical activities could modify gut microbiota [4,5]. Recent evidences suggest that a useful method for assessing the alteration of intestinal permeability is the dosage of Zonulin in the patient's stools [6]. Thus, the aim of this study was to evaluate the role of chronic exercise on the gut flora composition and intestinal permeability in patients with stable T2D.

\section{Materials and Methods}

This research was a controlled open-label trial. Research protocol was approved by the Ethics Committee of Spedali Civili di Brescia, and performed in accordance with the Declaration of Helsinki. We selected non-smokers 15 males patients with mean age of $69 \pm 1.3$ years with a controlled diet of 7949kJ (1900 


\section{Current Research in Diabetes \& Obesity Journal}

kcal) derived from 40-60\% carbohydrates, 30\% fat and 10-20\% protein. About $20 \mathrm{~g} / 1000 \mathrm{kcal}$ of fibre were present.

Patients had diagnosis of T2D for at least 2 years, no need for insulin therapy, arterial hypertension and dyslipidemia controlled by statins and either ACE-inhibitors or angiotensin receptor blockers, absence of diabetes-specific complications and/or ischemic heart disease and ability to perform physical activities. Patients with endocrine disorders and inflammatory or malabsoptive intestinal diseases were not included in the study. Patients studied did not used antibiotic, steroid, laxative, antidiarrheal and/or probiotic treatment over the previous 3 months and during the study. Biochemical measurements and gut flora was determined as we described before [7]. Measurements of biochemical variables, including C-reactive protein (CRP) as marker of systemic inflammation, were performed in peripheral venous sample after 12-h overnight fast.

Gut Flora were determined in stool sample collected with strikers and inserted in a hermetic vials with a specific medium. Then, microbiota were counted after $48 \mathrm{~h}$ of incubation at proper condition with a selective agar. A further proof of the isolation was performed with bacterial metabolic tests performed on isolated organisms through "BBL Crystal Identification System" (Becton Dickinson NJ USA). The results are expressed in CFU (Colony Forming Units)/ml of stool. The test was performed by laboratory of clinical and virology Functional Point (Bergamo. Italy) which follows international standard of quality control and it is accredited with the national health system. The tests reproducibly was $<9 \%$. LIS was measured as faecal Zonulin concentration $(\mathrm{ng} / \mathrm{ml})$ using a commercial ELISA kits (Immunodiagnostic AG, Bensheim, Germany).

T2D patients were treated with standard medical care. They have optimal glycaemic, lipid, blood pressure and body weight targets according to the international guidelines. The training program was of six months of endurance, resistance and flexibility training, following the most recent guidelines of the Italian Society of Diabetology and Medical Diabetology Association as described in details elsewhere [8]. Breathy, endurance training involved cycling on mechanically braked cycle ergometers while wearing heart rate monitors, at the intensity individually prescribed according to the baseline results of the exercise test. Time of exercise has been increased progressively in the first 3 months, starting from 15 minutes and reaching the target of 35 minutes.

Resistance training was of 40 to 50 minutes of different exercises which involved the major muscle groups (upper limb, lower limb, chest, back and core). Exercises included both calisthenics and repetitions with ankle weights, dumbbells and elastic bands. Patients started with 3 sets of 8 repetitions, then progressively improved to 3 sets of $12-15$ repetitions. Flexibility training was of static stretching exercises that involved upper and lower body, performed before and after the resistance training exercise. All training sessions were performed in a hospital-based setting and under the supervision of specialized personal. Exercises was performed 3-time a week of about 90 minutes each section. To assess the statistical significance of differences between the variables measured before starting the excise programs (baseline $=\mathrm{T} 0$ ) and after 6 months of exercise training (T1), we used Student's t-test for paired samples. A value of $\mathrm{p}<0.05$ was considered statistically significant.

\section{Results}

As expected, at baseline (TO), T2D patients showed altered biochemical parameters (glycaemia, HOMA index, HbA1c, CRP) (Table 1). In addition, these patients had dysbiosis with little reduction of Lactobacillus and presence of pathogenic gut flora as documented by the high concentration of Mycetes spp. In addition, T2D patients showed higher Zonulin concentration in the stool suggesting altered intestinal impermeability (Table 2). Six mounts of exercise training (T1) improved glycaemia, HOMA index and HbA1c in T2D patients. Interestingly, exercise attenuated systemic inflammation measured as CRP also (Table 1). Furthermore, chronic exercise increased the intestinal concentration of Lactobacillus and significantly reduced Mycetesspp and Zonulin concentration (Table 2).

Table 1: Biochemical measurements before (T0) and after exercise training (T1). Data are expressed as mean \pm sd.

\begin{tabular}{|c|c|c|c|}
\hline & T0 & T1 & p \\
\hline $\begin{array}{c}\text { Glucose (mg/ } \\
\text { dl) (nv=70- } \\
\text { 100) }\end{array}$ & $139 \pm 10.1$ & $129 \pm 9.5$ & $<0.05$ \\
\hline $\begin{array}{c}\text { HbA1c (\%) } \\
\text { (nv=4-6) }\end{array}$ & $7.0 \pm 0.2$ & $5.8 \pm 0.3$ & $<0.05$ \\
\hline $\begin{array}{c}\text { HOMA index } \\
\text { (nv=0.22-2.5) }\end{array}$ & $4.58 \pm 0.6$ & $3.52 \pm 0.7$ & $<0.05$ \\
\hline $\begin{array}{c}\text { CRP(mg/dl) } \\
\text { (nv=0-5) }\end{array}$ & $6.1 \pm 1.0$ & $9.1 \pm 1.3$ & $<0.05$ \\
\hline
\end{tabular}

$\mathrm{nv}=$ Normal Value

Table 2: Gut flora composition (Genus/Species-x 105cfu/ml) and Zomuline $(\mathrm{ng} / \mathrm{ml})$ faecal concentration before (T0) and after exercise training(T1). Data are expressed as mean \pm sd.

\begin{tabular}{|c|c|c|c|}
\hline & T0 & T1 & p \\
\hline Lactobacillusspp $(\mathrm{nv}>150)$ & $99.8 \pm 39.4$ & $119 \pm 48.9$ & $\mathrm{~ns}$ \\
\hline $\begin{array}{c}\text { Bifidobacteriumspp } \\
(\mathrm{nv}>200)\end{array}$ & $220.5 \pm 52.3$ & $246.6 \pm 69.1$ & $\mathrm{~ns}$ \\
\hline Enterococcusspp $(\mathrm{nv}<150)$ & $0.6 \pm 2.2$ & 0 & $\mathrm{~ns}$ \\
\hline Streptococcusspp $(\mathrm{nv}<150)$ & 0 & 0 & $\mathrm{~ns}$ \\
\hline Bacteroidesspp $(\mathrm{nv}>150)$ & $162.1 \pm 33.3$ & $185.0 \pm 56$ & $\mathrm{~ns}$ \\
\hline E. Coli $(\mathrm{nv}<150)$ & $160.1 \pm 56.6$ & $138.0 \pm 63.0$ & $\mathrm{~ns}$ \\
\hline Candidaspp $(\mathrm{nv}=0)$ & $8.0 \pm 9.2$ & $4.6 \pm 5.0$ & $\mathrm{~ns}$ \\
\hline Mycetes $(\mathrm{nv}=0)$ & $310 \pm 42$ & $191.5 \pm 45$ & $<0.001$ \\
\hline Campylobacterspp $(\mathrm{nv}=0)$ & $2.0 \pm 3.2$ & $3.2 .7 \pm 8.1$ & $\mathrm{~ns}$ \\
\hline Clostridium Difficile $(\mathrm{nv}=0)$ & $0 \pm 1.5$ & $0.1 \pm 0.8$ & $\mathrm{~ns}$ \\
\hline
\end{tabular}




\section{Current Research in Diabetes \& Obesity Journal}

\begin{tabular}{|c|c|c|c|}
\hline Salmonellaspp $(\mathrm{nv}=0)$ & $5.0 \pm 5.2$ & $6.2 \pm 5.9$ & $\mathrm{~ns}$ \\
\hline Shigellaspp $(\mathrm{nv}=0)$ & $4.1 \pm 1.4$ & $3.5 .3 \pm 2$ & $\mathrm{~ns}$ \\
\hline $\begin{array}{c}\text { Yersinia Enterocolitica }(\mathrm{nv} \\
=0)\end{array}$ & $3 \pm 0.7$ & $2.2 .1 \pm 1.2$ & $\mathrm{~ns}$ \\
\hline $\begin{array}{c}\text { Zonuline }(\mathrm{nv}<60) \\
\text { Z }\end{array}$ & $212+36$ & $79+25$ & $\mathrm{P}<0.001$ \\
\hline
\end{tabular}

$\mathrm{nv}=$ Normal Value

\section{Discussion}

This pilot study showed that selected patients with T2D had intestinal dysbiosis with overgrow of mycetes, presence of "Leaky gut syndrome" and chronic inflammation. Interestingly, chronic exercise significantly reduced all these parameters. Previous studies show that dysbiosis is present in T2D patients $[9,10]$. In line with these data, we found a massive presence of mycetes and candida in T2D patients, and inflammatory index. It is well known that gut mycetes stimulate systemic inflammation. Indeed, mycetes activates the innate immune receptor C-Type Lection Dectin-1. In detail, Dectin-1 is a cell receptor which reacts with B-1,3-glucans which is presents in the fungi wall. In turns, Dection-1 stimulates intracellular caspase recruitments domain-containing protein 9 with consequent local and generalised activation of inflammation due to inflammatory cytokine production and consequent stimulation of $\mathrm{T}$ helper 17 [11]. Notably, systemic inflammation is present in diabetic patients and it is consider one of the possible pathophysiological cause of this metabolic syndrome [2]. In addition, local intestinal inflammation could induce altered intestinal importability with consequent loss of gut fundamental functions. Indeed, for the first time in these patients, we found increased Zonulin's faecal concentration suggesting the presence of "Leak gut syndrome". Indeed, Zonilin is the proteins that physiologically modulates the intracellular intestinal cells tight junctions [6]. Traditionally, the functions of intestinal tract is the digestion and abortions of the ingested nutrients. However, recent evidences show that intestine regulates the immune and endocrine system by producing specific inflammatory molecules and/or hormones. In addition, it regulates the trafficking of macromolecules and/or microorganism between intestinal lumen and blood influencing systemic inflammation. It is intuitive that maintenance of intestinal impermeability and functions is crucial to maintain global metabolic body homeostasis avoiding the presence of "Leak gut syndrome" and the consequence malfunction.

It is known that exercise controls glycaemia and inflammation but here, for the first time, we showed that exercise decreases gut mycetes colonisation and the presence of "Leak gut syndrome" in T2D patients, likely improving important intestinal functions. The mechanisms by which exercise modified gut flora and reduced is not known yet. Recent data shows that exercise influences microbiota by several mechanisms. Indeed, exercise may modify bile acids profiles [12] and/or faecal short chain fatty acids (SCFAs) as butyrate [13]. Exercise may also interact with gut immunological function increasing intestinal immunoglobulin A (IgA), decreasing number of lymphocytes-B and CD4+T cells, and influencing gene expression of cytokines as IL-6, Il-4, IL-10 and TGF-B [14]. Exercise can also modify microbiota because is able to reduce intestinal transit time [15].

We think that our data, although preliminary, could have important clinical implications. Indeed, for the first time, we showed that patients with T2D have heavy intestinal mycetes colonisation and LIS, and chronic exercise can reduced these alteration. This likely could improving intestinal function which influence nutrients metabolism, hormonal production and absorption of oral drug-administered. So, exercise, with or without a specific therapies able to cure of intestinal microbiota, could be an important step for tailored therapy allowing traditional therapy and patients metabolism to function more properly.

This study has some limitations. We used a selective culture medium to identify bacteria and mycetes instead of molecular biology techniques. Indeed, we don't want to provide a "faecal finger print" of patients. We won't identify saprophytes and some minor intestinal pathological and mycetes species capable of stimulating inflammation without gastrointestinal symptoms [7]. This is a pilot study with a limited number of selected patients. We have in progress a large scale study to confirm these results.

\section{References}

1. Wing RR, Bolin P, Brancati FL, Bray GA, Clark JM, et al. (2013) Cardiovascular effects of intensive lifestyle intervention in type 2 diabetes. N Engl J Med 369(2): 145-154.

2. Cani P, Amar J, Iglesias M, Poggi M, Knauf C, et al. (2007) Metabolic endotoxemia initiates obesity and insulin resistance. Diabetes 56(7): 1761-1772.

3. Groschowitz K, Hogan S (2009) Intestinal barrier function: molecular regulation and disease pathogenesis. J Allergy Clin Immunol 124(1): 3-20.

4. Clarke SF, Murfhy EF, O’Sallivan O, Lucey AJ, Humphreys M, et al. (2014) Exercise and associated diatary extremes impact on gut micorbial diversity. Gut 63(12): 1913-1920.

5. Cerdà B, Perez M, Perez Santiago JD, Tornero Aguilera JF, González Soltero R, et al. (2016) Gut micorbiota modification: another piece in the puzzle of the benefits of physical exercise in health? Front Physiol 7: 51 .

6. Fasano A (2011) Zonulin and its regulation of intestinal barrier function: the biological door to inflammation, autoimmunity, and cancer. Physiol Rev 91(1): 151-175.

7. Pasini E, Aquilani R, Testa C, Baiardi P, Angioletti S, et al. (2016) Pathogenic gut flora in patients with chronic heart failure. JACC Heart Fail 4(3): 220-227.

8. Vinetti G, Mozzini C, Desenzani P, Boni E, Bulla L, etal. (2015) Supervised exercise training reduces oxidative stress and cardiometabolic risk in adults with type 2 diabetes: a randomized controlled trial. Sci Rep 5: 9238. 


\section{Current Research in Diabetes \& Obesity Journal}

9. He C, Shan Y, Song W (2015) Targeting gut microbiota as a possible therapy for diabetes. Nutr Res 35(5): 361-367.

10. Gosiewski T, Salamon D, Szopa M, Sroka A, Malecki MT, et al. (2014) Quantitative evaluation of fungi of the genus Candida in the feces of adult patients with type 1 and 2 diabetes - a pilot study. Gut Pathog 6(1): 43.

11. Iliev ID, Funari VA, Taylor KD, Nguyen Q, Reyes CN, et al. (2012) Interaction between commensal fungi and the C-Type lection receptor Dection-1 influence colitis. Science 336(6086): 1314-1317.

12. Wertheim BC, Martínez ME, Ashbeck EL, Roe DJ, Jacobs ET, et al. (2009) Physical activity as a determinant of fecal bile acid levels. Cancer Epidemiol Biomarkers Prev 18(5): 1591-1598.

This work is licensed under Creative Commons Attribution 4.0 Licens

DOI: 10.19080/CRDOJ.2017.05.555659
13. Matsumoto M, Inoue R, Tsukahara T, Ushida K, Chiji H, et al. (2008) Voluntary running exercise alters microbiota composition and increases n-butyrate concentration in the rat cecum. Biosci Biotechnol Biochem 72(2): 572-576.

14. Viloria M, Lara Padilla E, Campos Rodríguez R, Jarillo Luna A, Reyna Garfias H, et al. (2011) Effect of moderate exercise on IgA levels and lymphocyte count in mouse intestine. Immunol Invest 40(6): 640-656.

15. Oettlé GJ (1991) Effect of moderate exercise on bowel habit. Gut 32(8): 941-944.

Your next submission with Juniper Publishers will reach you the below assets

- Quality Editorial service

- Swift Peer Review

- Reprints availability

- E-prints Service

- Manuscript Podcast for convenient understanding

- Global attainment for your research

- Manuscript accessibility in different formats ( Pdf, E-pub, Full Text, Audio)

- Unceasing customer service

Track the below URL for one-step submission https://juniperpublishers.com/online-submission.php 